\title{
Small Angle X-Ray Scattering Technique for the Particle Size Distribution of Nonporous Nanoparticles
}

\author{
A. Agbabiaka, ${ }^{1}$ M. Wiltfong, ${ }^{2}$ and C. Park $^{1}$ \\ ${ }^{1}$ Department of Industrial and Manufacturing Engineering, Florida State University, 2525 Pottsdamer Street, Tallahassee, FL 32310, \\ USA \\ ${ }^{2}$ North Carolina State University, Department of Mechanical \& Aerospace Engineering, 911 Oval Drive, Raleigh, NC 27695, USA
}

Correspondence should be addressed to C. Park; cpark5@fsu.edu

Received 22 July 2013; Revised 19 November 2013; Accepted 20 November 2013

Academic Editor: Frank Hubenthal

Copyright (C) 2013 A. Agbabiaka et al. This is an open access article distributed under the Creative Commons Attribution License, which permits unrestricted use, distribution, and reproduction in any medium, provided the original work is properly cited.

\begin{abstract}
Nanoparticles are small particles whose sizes are less than $100 \mathrm{~nm}$. They have many industrial applications due to their unique properties. Their properties are often size-dependent; thus the accurate determination of nanoparticle sizes is important for quality assurance of nanoparticle production processes. A small angle X-ray scattering technique is a promising method used for characterizing nanoparticle sizes. It has distinctive advantages over other techniques such as electron microscope techniques. In this paper, we review the state-of-the-art methods for determining the sizes of nanoparticles with small angle X-ray experiments and discuss the advantages and limitations of the state-of-the-art methods.
\end{abstract}

\section{Introduction}

Nanoparticles are tiny particles whose sizes are less than $100 \mathrm{~nm}$ and have many industrial applications due to their novel physical and chemical properties, nanobiotechnology [1], drug delivery [2,3], catalysis [4-7], fluorescent biological labels [8], biodetection of pathogens [9], chemical sensors [10], optical/electronic/magnetic devices [11], and medicine [12]. The physical and chemical properties exhibited by nanoparticles are often size-dependent [13-16]. Therefore, by controlling the size of nanoparticles, we should be able to draw the desired properties of the nanoparticles.

In order to control the particle sizes, we should be able to accurately quantify the sizes of the nanoparticles produced from a synthesis process of nanoparticles, because we cannot control what we cannot quantify. Therefore by quantifying the sizes of nanoparticles we can control its synthesis process and its production. The accurate size determination becomes even more important on the industry scale, because it helps install quality assurance of nanoparticle production processes [17, 18].
There are several techniques that obtain the size information of nanoparticles, atomic force microscopy (AFM) [19], transmission electron microscopy (TEM) [20], scanning electron microscopy (SEM) [21], differential mobility analysis, dynamic light scattering (DLS) [22], and small angle $\mathrm{X}$-ray scattering (SAXS). Depending on the material to be characterized, each of these techniques has its own peculiar advantages and disadvantages. The SAXS has the advantage over other techniques of being able to analyze a wide variety of sample types, including aerosols, colloidal suspensions, powders, solids, and thin films. Another advantage of the SAXS over the electron microscopy (EM) methods is that the SAXS often requires very little sample preparation time, in contrast to the elaborate sample preparation procedures required for using the electron microscopy (EM) methods. Compared to the EM methods, the SAXS tends to provide more statistically reliable estimates of nanoparticle sizes because the particle size distribution obtained by the SAXS is typically estimated over a large number of nanoparticles, while the size distribution obtained by the EM methods is based on the measurements of a few hundred or thousand 
particles. Furthermore, recent studies suggest that the SAXS can also be used for the in situ or online monitoring of nanoparticle systems [23-25] and for the determination of nanoparticle shapes [26].

A SAXS experiment typically produces a one-dimensional scattering intensity obtained by circular averaging of the $2 \mathrm{D}$ scattering pattern observed in the experiment, which has different patterns depending on the sizes and shapes of nanoparticles or their spatial arrangements. In order to determine the size information about nanoparticles from the intensity, we should perform a data analysis problem based on the theory of SAXS, which is well known as a nontrivial scientific inverse problem.

The objective of this review is to discuss the state-of-theart methods in analyzing the SAXS data to obtain the particle size distribution for a system of nanoparticles having different sizes, specifically for nonporous nanoparticles. We categorize the methods into four groups, depending on the underlying assumptions and the techniques applied: the average size determination method, the parametric distribution model method, the integral transform method, and the numerical method, which are summarized in Table 1.

The average size determination method is the simplest approach among all the categories [27-45]. It assumes that all of the nanoparticles in a system of interest are equally sized and shaped, and it determines the size of the identically shaped nanoparticles. When the system of interest is indeed monodispersed (i.e., all nanoparticles are identical in terms of size and shape), this method is simple and effective, but when the assumption is wrong, it only provides the average size of a population of nanoparticles in the system of interest. For more details, see Section 2.

The parametric distribution model method assumes that only the sizes of nanoparticles in a system vary, while their shape is still assumed to be the same, and also assumes that the particle size distribution is a certain parametric form [46-55]. The objective of the method is to determine the parameters of the parametric distribution function giving the best agreement between the predicted intensity of the system and the experimental intensity. For details, see Section 3.

The integral transform method makes no assumption on the form of the particle size distribution function, but the shape of the particles still needs to be assumed beforehand [56-70]. The size distribution function is directly obtained using an integral transform, generally known as the Titchmarsh transform which gives the size distribution as an integral function of the experimentally determined scattered intensity [70]. An advantage of the method is that users do not need any a priori knowledge on the form of a particle size distribution, which is in general hard to know before the analysis. However, the method is computationally difficult to solve due to the integral transform.

The numerical methods are based on the same assumptions of the integral transform method, but it is more computationally easy [71-94]. It is based on a certain discretization of a particle size distribution. This discretization process leads to a set of linear equations for the discretized size distribution function. Solving the linear equation is relatively simple using many available linear equation solvers.
All of the aforementioned categories of the methods have one common assumption that nanoparticles in a system are sparsely located, which we call a system of diluted nanoparticles. For such a system, the SAXS scattering intensity curve only depends on the particle size distribution. However, the assumption may not be true for some systems of nanoparticles, where nanoparticles are more densely spaced in a sample. For a sample of densely located nanoparticles, the scattering intensity curve does not only depend on the particle size distribution but also depends on the spatial arrangements of the nanoparticles in the sample. We also review how to extend the aforementioned methods with consideration of spatial arrangements of nanoparticles. For more details, see Section 6.

The rest of this paper is organized as follows. Each of Sections 2 through 5 describes the aforementioned four categories of the methods for particle size determination. Section 6 explains the methods of considering the spatial arrangements of nanoparticles in the SAXS data analysis for a system of concentrated nanoparticles. Section 7 concludes this paper with discussion.

\section{Determination of the Average Particle Size}

This approach is based on the assumption that a system of nanoparticles is monodispersed that is, all nanoparticles in the system are assumed to be identical in terms of their shapes and sizes. The objective of this approach is to determine the particle size and shape. There are two general approaches, the model-free approach and the direct modelling approach.

The model-free approach involves the determination of several geometrical parameters of nanoparticles directly from the experimentally measured intensity curve. These parameters give an overall understanding of the nanoparticles' geometries, which include the particle volume, the molecular mass of the particles, the forward scattering, the radius of gyration of the particle, the surface area per unit volume, the largest dimension of the particle, and the pair distance distribution function (PDDF) [27, 31]. These parameters are determined using the well-established theory of small angle $\mathrm{X}$-ray scattering, making use of the well-known physics laws such as the Guinier law, which gives an approximation of the initial portion of the scattering intensity by a simple exponential function, and the Porod law, which gives an approximation for the intensity curve for large scattering angles [27]. The determined parameters give simple numerical values, which characterizes the nanoparticles' geometries. The model-free approach is also very useful for characterizing a very complex structure such as complex aggregates of nanoparticles. One can determine the degree of aggregation, the fractal dimension, and the branched content based on the model-free approach [95].

It is often desirable to have a more detailed understanding of the particles beyond the simple parameters. When we need more detailed structural information of nanoparticles than what is obtainable by the model-free approach, the direct modeling approach is more appropriate. In these schemes, a theoretical geometry model is postulated to be the geometry 
TABLE 1: Summary of the small angle X-ray data analysis methods.

\begin{tabular}{|c|c|c|c|}
\hline Methods & Underlying assumptions & Advantages & Disadvantages \\
\hline $\begin{array}{l}\text { Average size } \\
\text { determination method } \\
(\mathrm{ADM}) \\
{[27-45]}\end{array}$ & $\begin{array}{c}\text { All nanoparticles in a system are } \\
\text { assumed to be equally sized; } \\
\text { determine the average size of } \\
\text { nanoparticles }\end{array}$ & $\begin{array}{l}\text { Straightforward and easy to } \\
\text { implement }\end{array}$ & $\begin{array}{c}\text { Typically nanoparticles have various } \\
\text { sizes and shapes }\end{array}$ \\
\hline $\begin{array}{l}\text { Parametric distribution } \\
\text { model (PDM) } \\
\text { [46-55] }\end{array}$ & $\begin{array}{l}\text { A parametric form of particle size } \\
\text { distribution is assumed; a particle } \\
\text { shape is typically fixed; a particle is } \\
\text { sparsely distributed in a sample }\end{array}$ & $\begin{array}{l}\text { Straight forward and easy to } \\
\text { implement }\end{array}$ & $\begin{array}{l}\text { For many cases, we do not have } \\
\text { whether a particle size distribution } \\
\text { follows a simple parametric form }\end{array}$ \\
\hline $\begin{array}{l}\text { Integral transform } \\
\text { method (ITM) } \\
{[56-70]}\end{array}$ & $\begin{array}{c}\text { The shape of particle is assumed; a } \\
\text { particle is sparsely distributed in a } \\
\text { sample }\end{array}$ & $\begin{array}{l}\text { Various forms of the particle size } \\
\text { distribution are considered }\end{array}$ & $\begin{array}{c}\text { It often involves complex numerical } \\
\text { integrations which may become } \\
\text { divergent or unstable }\end{array}$ \\
\hline $\begin{array}{l}\text { Numerical method } \\
\text { (NM) } \\
{[71-94]}\end{array}$ & $\begin{array}{c}\text { The shape of particle is assumed; a } \\
\text { particle is sparsely distributed in a } \\
\text { sample }\end{array}$ & $\begin{array}{l}\text { A finite approximation of the ITM } \\
\text { method; more computationally } \\
\text { feasible }\end{array}$ & $\begin{array}{l}\text { The approximation is often solved } \\
\text { by an optimization procedure, } \\
\text { which is nonlinear, so it is hard to } \\
\text { obtain the global optimal } \\
\text { approximation }\end{array}$ \\
\hline
\end{tabular}

of the nanoparticles, the theoretical intensity of the model is then computed using the SAXS theory, and the parameters of the model are iteratively updated until the best agreement between the model-based intensity and the experimental intensity is achieved. The model with the optimal parameters explains the geometry of nanoparticles.

A simple direct modeling approach is to approximate a particle's geometry by a simple geometrical shape such as a sphere, an ellipsoid, and a cylinder [27]. The subparticle method takes a combination of several simple geometrical shapes to model more complex geometrical shapes [32]. This method packs small spheres within the arbitrary shaped geometry and computes the theoretical intensity of the arbitrary shape by Debye's scattering formula. In the above methods, the dimensions of the geometrical models are optimized with respect to the model parameters that give the best possible agreement between the experimental intensity and the theoretically computed intensity.

Along this theme of geometric modeling of scattering intensities are methods based on the use of Monte Carlo procedures to calculate the theoretical scattering intensity of an arbitrary particle shape. In Hanson's paper, he describes a method used to build a complex model by using multiple, geometric building blocks (ellipsoids, cylinders, prisms, etc.) [33]. Briefly, these building blocks are spatially arranged with random coordinates, and the pair distance distribution function of the random coordinates is used to calculate the model intensity. The method is easy to implement but applies only to homogenous particles (i.e., particles having the same size and shape). Extension of the method to the general case of heterogenous particles [34] has been developed as well as to the case of anisotropic scattering (i.e., oriented particles) [35]. While each of the three methods is fast to implement, the use of random numbers to generate coordinates in the scattering volume must necessarily imply that the accuracy of the methods depends largely on the true quality of the random number generators used.
The $a b$ initio shape determination method is an alternative method of determining the particle shape from the analysis of X-ray scattering profiles for a monodispersed dilute solution of nanoparticles, which was initiated in 1971 by Stuhrmann [36]. It is based on the theory of multipole expansion using spherical harmonics. It differs from the above methods of geometrical shape modeling because it removes the trial and error process in the selection of models to fit to experimental data. Sturhmann's method takes advantage of the fact that the scattering intensity of a dilute solution of monodisperse particles is proportional to the scattering intensity of a single particle; thus the essential task for this type of solution is usually the shape determination or reconstruction of the particle or restoration of the $3 \mathrm{D}$ electron density in the particle system. The method starts by expanding the 3D surface of the shape of particle in terms spherical harmonics according to the following equation:

$$
F(\omega)=\sum_{l=0}^{l=L} \sum_{m=-l}^{m=l} f_{l m} Y_{l m}
$$

where $Y_{l m}$ 's are the standard mathematical functions known as the spherical harmonics and the numbers $f_{l m}$ are the coefficients of the multipole expansion which are easily obtained by integrating both sides of (1) for $f_{l m}(r)=\int F(\omega) Y_{l m}^{*} d \omega$ [37]. Using these expansions, it is possible to develop the scattering intensity of a closed geometrical shape in terms of the shape coefficients $f_{l m}$ as was performed by Stuhrmann [36]. The result is an infinite series representation of the scattering intensity of the shape enclosed by the bounded surface $F(\omega)$ shown below:

$$
I(s)=2 \pi^{2} \sum_{l=0}^{l=L} a_{n} s^{2 n},
$$

where $a_{n}$ are given in terms of the shape coefficients $f_{l m}$ and powers of the shape $f_{l m}^{(q)}$. 
However the practical implementation of the method using the infinite series above was hampered by the nonconvergence of the infinite series for larger scattering vector $s$ and by the difficulty of the numerical integration required for the calculation of the shape coefficients. However, in 1991, a breakthrough occurred in the field of ab initio shape determination using multipole expansion when Svergun and Stuhrmann improved the basic method of Sturmann by replacing the cumbersome and less accurate numerical integration with an analytical expression [38]. The shape coefficients $f_{l m}$ are then determined by evaluating the analytical expression in recursive manner. The values of $f_{l m}$ determine the particle shape. The uniqueness of the determined shape was addressed in Svergun's paper [39]. The method was extended to study the internal structures of a particle using the techniques of contrast variation [40]. The multipole theory of X-ray scattering has been extended by incorporating the group theory and the maximum entropy to better efficiently calculate scattering curves and reconstruct shapes [41]. The theory was extended to higher scattering angles [42]. Several other extensions of the theory have been considered such as the bead modeling [43], the dummy residue method [44], and the rigid body modelling [45].

The aforementioned methods assume that nanoparticles in a system are identical. However, many systems of nanoparticles in practice contain differently sized nanoparticles, whose system is called a polydispersed system. When the methods are applied to a polydispersed system, the methods are able to determine only the average size of the nanoparticles in a system. If one needs to obtain the actual particle size distribution, one should follow the model-based approach, which will be explained in the next section.

\section{Parametric Distribution Models}

The parametric distribution model method is the simplest method of the methods used in the determination of the size distribution of particles for a polydispersed system of nanoparticles. It is based on the assumption that all nanoparticles in a system have different sizes but the same known shape. The size of a nanoparticle is represented by a size parameter $R$. The size parameters for the nanoparticles in a system are assumed to follow a particle size distribution in a known parametric form, $D_{N}(R)$. The scattering intensity from such a system of nanoparticles is given as follows [27]:

$$
I(s)=\int_{0}^{\infty} i_{0}(s R) D_{N}(R) m^{2}(R) d R,
$$

where $s$ is the scattering vector, $i_{0}(s R)$ is a form factor for a particle of size $R$, and $m(R)$ relates the volume of a particle having size parameter $R$. The size distribution function $D_{N}(R)$ is parametric, which means that a few unknown parameters fully determine the distribution function, for example, the mean and standard deviation parameters in the normal distribution, and thus the theoretical scattering intensity $I(s)$ depends on the parameters via (3). The parameters giving the best agreement between the experimental scattering intensity and the theoretical scattering intensity are chosen by a general optimization algorithm to determine the size distribution function.

$I(s)$ was first approximated by the Guinier law with a certain assumed parametric distribution function such as the Maxwell distribution, the Schultz distribution, the Gaussian distribution, and the rectangular distribution, and the approximate $I(s)$ was fit to the experimental intensity curve for finding the distribution parameters [46]. The method was applied to study the particle size distribution of silicaalumina gels [47] and the particle size of gamma-alumina and carbon black [48]. Instead of using the approximate theoretical intensity, the exact form of the theoretical scattering intensity curve was derived for nanoparticles having a simple shape (spheres, rods, and plates) with the particle sizes following a parametric distribution $[49,50]$. The method then proceeded to find the values of the distribution parameters giving the best agreement with experiment. Similar results have been reported for nanoparticles having an ellipsoidal shape $[51,52]$. The scattering function of the ellipsoid with appropriate distribution function was used to express the theoretical SAXS intensity in terms of simple functions [53]. Another paper [54] used the log normal distribution as a particle size distribution, and the distribution was fit to the experimental intensity curve for determining the average size of nanoparticles and the particle size distribution, which was compared to the particle sizes observed from the TEM image analysis. The same approach was taken to determine the particle size distribution for nanostructured titanium oxide films [55].

All of the methods discussed in this section make the assumptions that the shapes of nanoparticles are the same, and the particle size distribution is in a known parametric form. However, the assumptions are not correct for some particular systems of nanoparticles. For example, in studies of inorganic systems, the size distribution of particles can hardly be expected to follow a particular form [27]. These problem necessitates the development of a whole new category of methods based on the direct inversion of (3) to get the size distribution function in terms of the scattering intensity without the need for prior assumption on the form of the distribution to be made. These methods will be considered in the next section.

\section{Integral Transform Methods}

The methods in the last section were all based on the assumptions of a certain parametric form of the particle size distribution. The methods discussed in this section make no assumption on the form of the size distribution function. The integral transform method directly inverts integral equation (3) for the particle size distribution [56]. The method assumes that nanoparticles are spherically shaped and gets an analytic expression for the size distribution of the particles, which involves an integral transform of the experimental scattering intensity curve. Similar studies $[57,58]$ have also been carried out each of which also involves different integral transforms of the scattering intensity. The integral transforms were numerically solved. However, these were rather theoretical 
studies, and no attempt was made to analyze real experimental scattering curves. Equation (4) was derived for estimating the particle size distribution for a system of polydispersed particles from the experimental intensity $I(s)$ [59]:

$$
D_{N}(R)=\frac{\text { const }}{R} \int_{0}^{\infty}\left(s^{4} I(s)-c_{4}\right) f(s, R) d s,
$$

where $f(s, R)$ is a known function of the size parameter $R$ and the scattering vector $s$ and $c_{4}$ is a constant obtained from the scattering intensity. The method was validated for its accuracy by running the method with experimental scattering intensity curves from a known size distribution function. It was reported that the discrepancy between the known distribution function and the distribution function calculated by (4) was less than $0.5 \%$ and in most cases, less than $0.1 \%$ [59]. An improvement of this method was carried out [60] which is based on an extrapolation of the scattering data beyond the maximum scattering angle at which data can be obtained. The integral transform methods were tested on Ludox 4 silica suspensions and gold sols to retrieve the particle size distribution, which was compared with the particle sizes observed from electron micrographs [61]. Another improvement of the analytical integral transform method was tried [62] to reduce termination errors (the inaccuracies associated with the integral transform). The integral transform method has also been used to calculate the diameter distribution of thin spherical shells [63], the diameter distribution of assemblies of long cylinders [64], the length distribution of assemblies of thin cylinders [65], the diameter distribution of thin circular disk [66], and the diameter distribution of helical structures [67]. Equation (4) was extended for the application to some nonspherical particles [68]. It was shown that if the form factor of a particle can be expressed in this general form

$$
\begin{gathered}
i_{0}(s R)=\frac{J_{v}^{2}(s R)}{(s R)^{\beta}}, \\
m^{2}(R) \approx R^{2 \alpha},
\end{gathered}
$$

where $J_{v}(x)$ is the Bessel function of the first kind and order $\nu$ and $\alpha$ is the effective number of dimension ( $\alpha=3$ for Globular particle, 2 for platelets and 1 for rods), (4) can be rewritten in a function of the generalized form factors. The form factors of several shapes (e.g., uniform sphere, long randomly oriented cylinder, and thin disk) can be represented in the form of (5) with different values of $\alpha$ and $\beta$. The analytical expression of the particle size distribution for nanoparticles having shapes corresponding to the generalized form factor is given by

$$
s^{n} I(s)=\int_{0}^{\infty} s J_{v}^{2}(s R) D_{N}(R) R^{m} d R,
$$

where $n=\beta+1$ and $m=2 \alpha-\beta-1$. The integral on the right hand side, well known as the Titchmarsh transform, has an inverse that gives the size distribution in terms of the intensity:

$$
R^{m} D_{N}(R)=\int_{0}^{\infty}\left[s^{n} I(s)-c_{n}\right] \psi(s R) d s,
$$

where $c_{n}=\lim _{s \rightarrow \infty}\left[s^{n} I(s)\right]$ and $\psi(x)$ is a function involving the derivative of the Bessel function of the first and second kind which is given in [27]. An improvement of this integral transform method is considered [69]. A numerical corrector method based on the Titchmarsh transform is used to determine the particle size distribution (PSD) from noisy data [70].

General drawbacks of the integral transform methods are their sensitivity to strong termination effects, which leads to artificial oscillations in the resulting size distribution and some difficulties in calculating the constant term in (8) which depends on an extrapolation of the scattering intensity to infinite angle.

\section{Numerical Methods}

The numerical method approximates the particle size distribution by a certain regularized form of a distribution function, which potentially reduced the termination errors. Some methods approximate the particle size distribution by a finite sum of $D_{N}\left(R_{j}\right)$ 's [71-73], where $D_{N}\left(R_{j}\right)$ is a proportion of particles having size parameter $R_{j}$ for $j$ in a finite. With the restricted form, the integral transform in (4) becomes a simple finite sum as

$$
I\left(s_{i}\right)=\sum_{j=1}^{n} D_{N}\left(R_{j}\right) i_{0}\left(s_{i} R_{j}\right) m^{2}\left(R_{j}\right) W_{j} \Delta R_{j},
$$

where the experimental intensities are assumed to be available for a finite number of scattering vectors $s_{i}$ for $i=$ $1, \ldots, N$. Let I denote a vector of the experimental intensities and let $\mathbf{D}$ denote a vector of $D_{N}\left(R_{j}\right)$ 's. We obtain a linear system of equation for $\mathbf{D}$ :

$$
\mathrm{AD}=\mathbf{I},
$$

where $A_{i j}=i_{0}\left(s_{i} R_{j}\right) m^{2}\left(R_{j}\right) W_{j} \Delta R_{j}$. There are many versions of the methods that obtain the discretized distribution function $\mathbf{D}$. Some of the methods put a certain constraint on $\mathbf{D}$ to obtain a physically valid distribution function. For example, the nonnegativity constraint is imposed on $\mathbf{D}$ to get a solution [71]. Tikhonov's classical regularization was also used with a regularization parameter [72]; find general information for Tikhonov's regularization [73].

A different strategy for approximating the size distribution function is known as the indirect transform method (ITM) [74]. The method is based on the idea of representing the distribution function $D_{N}(R)$ by a superposition of orthogonal functions known as cubic splines:

$$
D_{N}(R)=\sum_{v=1}^{m} c_{v} B_{v}(R),
$$

where the coefficients of the summation $c_{v}$ are determined using the algorithm. Other regularization method for obtaining the size distribution function has been considered. The similar method has been proposed by Svergun et al. [75] and has been applied to determine the size distribution of mesoscopic metallic systems [76]. 
A variant of the original ITM method uses trigonometric functions [77] in the place of cubic spline functions $B_{v}(R)$ in (11). Another variant of the indirect transformation method is the maximum entropy method (MEM) $[78,79]$. The MEM method is based on the original idea of the maximum entropy developed for image reconstruction [80]. It was first applied to the small angle scattering experiment [81]. Its accuracy is comparable with the original method of Glatter [82] and the Bayesian analysis method [83]. The maximum entropy method has been tested on both simulated data $[84,85]$ and real experimental data [86].

The structure interference method (SIM) [87, 88] has a certain distinctive feature from the indirect transform method. It has been reported that the method has a certain advantage over the ITM method with respect to termination errors, influence of solution by oscillations, robustness, and real space resolution [88].

The Monte Carlo fitting (MCF) $[89,90]$ represents the particle size distribution as a finite number of spherical nanoparticles having random sizes, which was first applied to the wide angle X-ray scattering [91]. The algorithm iteratively adds more spherical particles having random sizes such that the resulting particle size distribution has a better agreement with experimental results. A variant of the method [92] uses a fixed number of spheres and instead continually changes the radii of selected spheres to better approximate experimental intensity. Several other Monte Carlo fitting schemes for analyzing scattering data have been proposed [93, 94].

\section{Consideration of Structure Factor and Multiple Scattering Effects}

The methods discussed in the previous sections have been based on a common underlying assumption that a system of nanoparticles being investigated is dilute, and therefore the effect of the spatial arrangement of nanoparticle to the small angle scattering intensity is negligible. In addition, the effect of multiple scattering is assumed to be negligible. These assumptions do not hold for the system of concentrated nanoparticles in which the spatial arrangement of nanoparticles affects significantly the measured scattering intensity $[96,97]$, particularly a portion of the intensity curve for small scattering vectors, and the multiple scattering affects the intensity significantly. Therefore, ignoring the spatial arrangement and the multiple scattering could lead to a wrong analysis.

The effect of multiple scattering is analytically studied in the literature $[98,99]$. The analytical form of the effect can be studied to estimate the effect of multiple scattering and correct the observed scattering intensity before the analysis of particle size distribution.

The spatial arrangement of nanoparticles in a system is typically considered by including a structure factor into the analysis of the scattered intensity. The structure factor is a function $S(s)$ depending on the spatial arrangement of nanoparticles in a system. Multiplying the structural factor with the theoretical intensity for a dilute system, it gives the theoretical intensity for a system of concentrated nanoparticles.

For a particular case of a monodispersed system, the scattering intensity is given as

$$
\begin{aligned}
I(s) & =N F^{2}(s) S(s) \\
& =N F^{2}(s)\left[1+\frac{N}{V} \int_{0}^{\infty} 4 \pi r^{2} d r(P(r)-1) \frac{\sin s r}{s r}\right],
\end{aligned}
$$

where $F(s)$ is the form factor corresponding to an assumed shape of nanoparticle, $V$ is the volume of a system, $N$ is the number of the particles within the volume, $S(s)$ is the structure factor depending on the spatial arrangement of nanoparticles, and $P(r)$ is the pair correlation function, which characterizes the pair distance function among the nanoparticles in a system. The liquid state theory $[100,101]$ is used for calculating the correlation function under certain assumptions of various nanoparticle interaction potential and closure relations that relate the interaction potentials directly to the direct correlation function. Some of the widely used interaction potentials are the hard sphere potential, the sticky hard sphere [102], the Yukawa potential (a.k.a. the screened coulomb potential), the DLVO potential [103], and the square-well potential. The closure relation between the correlation function and the potential is usually based on various heuristic physical arguments and approximations, and the common ones are the Percus-Yevick [104], the meanspherical approximation, the hypernetted chain, and the Roger-Young (RY). With a choice of the potentials together with a choice of the closure relation, the Ornstein-Zernike integral equation can be solved to yield the correlation function, and thus the structure factor is calculated using the integral expression in (12) above. The Ornstein-Zernike integral equation was solved to get a closed form analytic expression for the structure factor using the MSA closure and the Yukawa potential [105].

For polydispersed systems with size and shape polydispersity, individual particle sizes and shapes as well as their spatial arrangement affect the structure factor. The mixed effect is summarized to the effective structure factor [106]. For polydispersed spherical particles, the effective structure factor has been calculated and is given in terms of the partial structure factors, using the hard sphere potential and the Percus-Yevick approximation [107]. A solution of the Percus-Yevick approximation using the sticky hard sphere model for polydispersed particles was obtained [108], while a solution of the Ornstein-Zernike equation, under the mean square approximation for a polydispersed system of particles interacting in a Yukawa force field, was obtained [109]. Some of the other schemes that consider the effects of polydispersity on the structure factor are the decoupling approximation [110], which assumes no correlation between particle size and particle orientation, and the local monodispersed approximation [111] assuming that interactions only occur in between identical spheres. For nonspherical particles, the structure factors and their deviation from the case of spherical particles have also been studied $[112,113]$. The various expressions for 
the calculation of the effective structure factors have been incorporated into general fit procedures used to calculate scattering intensities. For example, the generalized indirect transform method (GIFT) is developed as an extension of the ITM discussed in Section $3[106,114]$ to include appropriate structure factors for both spherical and nonspherical particles. The applications of the GIFT method for aqueous nanocrystalline cellulose suspensions [115] and for a mixture of microemulsions [116] have been demonstrated. A binary mixture of charged colloidal solution has been also investigated [117]. The local monodispersed approximation has been applied to study silver nanoparticles [118], where the estimated size distribution was comparable with that obtained from the TEM image analysis of the same sample.

\section{Conclusion}

We reviewed a wide variety of the available methods for analyzing the small angle $\mathrm{X}$-ray scattering data of a polydispersed nanoparticle system to yield its particle size distribution. The choice of the method to be used for analysis would necessarily have to depend on several factors such as the easiness of implementation, prior knowledge for the form of the particle size distribution, the degree of polydispersity of a given sample, and the degree of particle concentration. When the system is known to contain spherically shaped particles of the same size, the simple methods in Section 2 will be appropriate. In the case that the particle size distribution can be expected to be in a simple parametric form, then the methods described in Section 3 would be the best approach. When there is no prior knowledge on the form of the size distribution, the indirect transform methods in Section 4, involving a certain integral transform without any assumption on the form, may be the better choice. The numerical methods in Section 5 will provide more computationally easier alternatives than the indirect transform methods, which are able to retrieve the particle size distribution of relatively complex size distribution functions and which are easier to implement than the integral transform methods. In the case of concentrated particle systems, we should consider the effect of the spatial arrangements of nanoparticles in a system onto a scattering intensity curve, and the structure factor must be calculated and included with the scattering intensity calculations. The generalized indirect transform method mentioned in Section 6 incorporates the structural factor into the integral transform method and would be the method of choice to determine the particle size distribution.

As we discussed, the small angle $\mathrm{X}$-ray scattering analysis provides an effective option of determining the particle size distribution for various types of nanoparticle systems. However, it is still recommended that the particle size distribution determined by one of the small angle X-ray scattering data analysis methods should be compared with the one determined by other techniques such as transmission electron microscope and dynamic light scattering for validation, especially for the cases requiring high degree of accuracy.

\section{References}

[1] O. V. Salata, "Applications of nanoparticles in biology and medicine," Journal of Nanobiotechnology, vol. 2, article 3, pp. 1-6, 2004.

[2] D. Pantarotto, C. D. Partidos, J. Hoebeke et al., "Immunization with peptide-functionalized carbon nanotubes enhances virusspecific neutralizing antibody responses," Chemistry \& Biology, vol. 10, no. 10, pp. 961-966, 2003.

[3] C. Mah, T. J. Fraites Jr., I. Zolotukhin et al., "Improved method of recombinant AAV2 delivery for systemic targeted gene therapy," Molecular Therapy, vol. 6, no. 1, pp. 106-112, 2002.

[4] T. J. Schmidt, M. Noeske, H. A. Gasteiger et al., "Electrocatalytic activity of PtRu alloy colloids for $\mathrm{CO}$ and $\mathrm{CO} / \mathrm{H}_{2}$ electrooxidation: stripping voltammetry and rotating disk measurements," Langmuir, vol. 13, no. 10, pp. 2591-2595, 1997.

[5] M. T. Reetz, R. Breinbauer, P. Wedemann, and P. Binger, "Nanostructured nickel-clusters as catalysts in [3+2] cycloaddition reactions," Tetrahedron, vol. 54, no. 7, pp. 1233-1240, 1998.

[6] M. T. Reetz, R. Breinbauer, and K. Wanninger, "Suzuki and Heck reactions catalyzed by preformed palladium clusters and palladium/nickel bimetallic clusters," Tetrahedron Letters, vol. 37, no. 26, pp. 4499-4502, 1996.

[7] A. Balamurugan, K.-C. Ho, and S.-M. Chen, "One-pot synthesis of highly stable silver nanoparticles-conducting polymer nanocomposite and its catalytic application," Synthetic Metals, vol. 159, no. 23-24, pp. 2544-2549, 2009.

[8] M. Bruchez Jr., M. Moronne, P. Gin, S. Weiss, and A. P. Alivisatos, "Semiconductor nanocrystals as fluorescent biological labels," Science, vol. 281, no. 5385, pp. 2013-2016, 1998.

[9] R. L. Edelstein, C. R. Tamanaha, P. E. Sheehan et al., "The BARC biosensor applied to the detection of biological warfare agents," Biosensors and Bioelectronics, vol. 14, no. 10-11, pp. 805-813, 2000.

[10] R. Elghanian, J. J. Storhoff, R. C. Mucic, R. L. Letsinger, and C. A. Mirkin, "Selective colorimetric detection of polynucleotides based on the distance-dependent optical properties of gold nanoparticles," Science, vol. 277, no. 5329, pp. 1078-1081, 1997.

[11] G. Schön and U. Simon, "A fascinating new field in colloid science: small ligand-stabilized metal clusters and their possible application in microelectronics-part II: future directions," Colloid \& Polymer Science, vol. 273, no. 3, pp. 202-218, 1995.

[12] I. Brigger, C. Dubernet, and P. Couvreur, "Nanoparticles in cancer therapy and diagnosis," Advanced Drug Delivery Reviews, vol. 54, no. 5, pp. 631-651, 2002.

[13] J. Baltrusaitis, P. M. Jayaweera, and V. H. Grassian, "Sulfur dioxide adsorption on $\mathrm{TiO}_{2}$ nanoparticles: influence of particle size, coadsorbates, sample pretreatment, and light on surface speciation and surface coverage," Journal of Physical Chemistry C, vol. 115, no. 2, pp. 492-500, 2011.

[14] A. Kasuya, G. Milczarek, I. Dmitruk et al., "Size- and shapecontrols and electronic functions of nanometer-scale semiconductors and oxides," Colloids and Surfaces A, vol. 202, no. 2-3, pp. 291-296, 2002.

[15] A. J. Maira, K. L. Yeung, C. Y. Lee, P. L. Yue, and C. K. Chan, "Size effects in gas-phase photo-oxidation of trichloroethylene using nanometer-sized $\mathrm{TiO}_{2}$ catalysts," Journal of Catalysis, vol. 192, no. 1, pp. 185-196, 2000.

[16] H. Yano, J. Inukai, H. Uchida et al., "Particle-size effect of nanoscale platinum catalysts in oxygen reduction reaction: an electrochemical and195Pt EC-NMR study," Physical Chemistry Chemical Physics, vol. 8, no. 42, pp. 4932-4939, 2006. 
[17] J. D. Morse, "Research challenges for integrated systems nanomanufacturing: report from the National Science Foundation Workshop," Internano 2008.

[18] C. Li, "Structure controlling and process scale-up in the fabrication of nanomaterials," Frontiers of Chemical Engineering in China, vol. 4, no. 1, pp. 18-25, 2010.

[19] J. Grobelny, F. W. DelRio, N. Pradeep, D.-I. Kim, V. A. Hackley, and R. F. Cook, "Size measurement of nanoparticles using atomic force microscopy," Methods in Molecular Biology, vol. 697, pp. 71-82, 2011.

[20] W. D. Pyrz and D. J. Buttrey, "Particle size determination using TEM: a discussion of image acquisition and analysis for the novice microscopist," Langmuir, vol. 24, no. 20, pp. 11350-11360, 2008.

[21] S. Sahoo, C. K. Chakraborti, S. C. Mishra, and U. N. Nanda, "Scanning electron microscopy as an analytical tool for particle size distribution and aspect ratio analysis of ciprofloxacin muco adhesive polymeric Suspension," Ijrras, vol. 6, no. 1, pp. 94-100, 2011.

[22] T. Walther, Photon Correlation Spectroscopy in Particle Sizing, John Wiley \& Sons, Chichester, UK, 2000.

[23] H. K. Kammler, G. Beaucage, D. J. Kohls, N. Agashe, and J. Ilavsky, "Monitoring simultaneously the growth of nanoparticles and aggregates by in situ ultra-small-angle X-ray scattering," Journal of Applied Physics, vol. 97, no. 5, Article ID 054309, 11 pages, 2005.

[24] M. Harada, N. Tamura, and M. Takenaka, "Nucleation and growth of metal nanoparticles during photoreduction using in situ time-resolved SAXS analysis," Journal of Physical Chemistry C, vol. 115, no. 29, pp. 14081-14092, 2011.

[25] L. C. McKenzie, P. M. Haben, S. D. Kevan, and J. E. Hutchison, "Determining nanoparticle size in real time by small-angle Xray scattering in a microscale flow system," Journal of Physical Chemistry C, vol. 114, no. 50, pp. 22055-22063, 2010.

[26] H. Brumberger, Modern Aspects of Small Angle Scattering, NATO Science Series C, Springer, New York, NY, USA, 1994

[27] L. A. Feigin and D. I. Svergun, Structural Analysis by Small Angle $X$-Ray and Neutron Scattering, Platinum Press, New York, NY, USA, 1987.

[28] I. Pilz, O. Glatter, and O. Kratky, "Small-angle X-ray scattering," Methods in Enzymology, vol. 61, pp. 148-249, 1979.

[29] B. Chu and B. S. Hsiao, "Small-angle X-ray scattering of polymers," Chemical Reviews, vol. 101, no. 6, pp. 1727-1761, 2001.

[30] R. Bienert, F. Emmerling, and A. F. Thünemann, "The size distribution of 'gold standard' nanoparticles," Analytical and Bioanalytical Chemistry, vol. 395, no. 6, pp. 1651-1660, 2009.

[31] O. Glatter, "The interpretation of real-space information from small-angle scattering experiments," Journal of Applied Crystallography, vol. 12, no. 2, pp. 166-175, 1979.

[32] J. J. Müller, G. Damaschun, and G. Hübner, "Small angle Xray scattering studies on the structure and symmetry of yeast pyruvate decarboxylase in solution," Acta Biologica et Medica Germanica, vol. 38, no. 1, pp. 1-10, 1979.

[33] S. Hansen, "Calculation of small-angle scattering profiles using Monte Carlo simulation," Journal of Applied Crystallography, vol. 23, no. 4, pp. 344-346, 1990.

[34] S. J. Henderson, "Monte Carlo modeling of small-angle scattering data from non-interacting homogeneous and heterogeneous particles in solution," Biophysical Journal, vol. 70, no. 4, pp. 1618-1627, 1996.
[35] B. C. McAlister and B. P. Grady, "Simulation of small-angle Xray scattering from single-particle systems," Journal of Applied Crystallography, vol. 31, no. 4, pp. 594-599, 1998.

[36] H. B. Stuhrmann, "Ein neues Verfahren zur Bestimmung der Oberflächenform und der inneren Struktur von gelösten globulären Proteinen aus Röntgenkleinwinkelmessungen," Zeitschrift für Physikalische Chemie, vol. 72, pp. 177-198, 1970.

[37] A. R. Edmonds, Angular Momentum in Quantum Mechanics, Princton University Press, 1996.

[38] D. I. Svergun and H. B. Stuhrmann, "New developments in direct shape determination from small-angle scattering. 1. Theory and model calculations," Acta Crystallographica Section $A$, vol. 47, no. 6, pp. 736-744, 1991.

[39] D. I. Svergun, V. V. Volkov, M. B. Kozin, and H. B. Stuhrmann, "New developments in direct shape determination from smallangle scattering. 2. Uniqueness," Acta Crystallographica Section A, vol. 52, no. 3, pp. 419-426, 1996.

[40] D. I. Svergun, V. V. Volkov, M. B. Kozin, H. B. Stuhrmann, C. Barberato, and M. H. J. Koch, "Shape determination from solution scattering of biopolymers," Journal of Applied Crystallography, vol. 30, no. 5, pp. 798-802, 1997.

[41] F. Spinozzi, F. Carsughi, and P. Mariani, "Particle shape reconstruction by small-angle scattering: integration of group theory and maximum entropy to multipole expansion method," The Journal of Chemical Physics, vol. 109, no. 23, pp. 10148-10158, 1998.

[42] V. L. Shneerson and D. K. Saldin, "Molecular shapes from small-angle X-ray scattering: extension of the theory to higher scattering angles," Acta Crystallographica Section A, vol. 65, no. 2, pp. 128-134, 2009.

[43] P. Chacón, F. Morán, J. F. Díaz, E. Pantos, and J. M. Andreu, "Low-resolution structures of proteins in solution retrieved from X-ray scattering with a genetic algorithm," Biophysical Journal, vol. 74, no. 6, pp. 2760-2775, 1998.

[44] D. I. Svergun, M. V. Petoukhov, and M. H. J. Koch, "Determination of domain structure of proteins from X-ray solution scattering," Biophysical Journal, vol. 80, no. 6, pp. 2946-2953, 2001.

[45] D. I. Svergun, "Mathematical methods in small-angle scattering data analysis," Journal of Applied Crystallography, vol. 24, no. 5, pp. 485-492, 1991.

[46] C. G. Shull and L. C. Roess, "X-ray scattering at small angles by finely-divided solids. I. General approximate theory and applications," Journal of Applied Physics, vol. 18, no. 3, pp. 295307, 1947.

[47] P. B. Elkin, C. G. Shull, and L. C. Roess, "Silica-Alumina, gels," Industrial \& Engineering Chemistry, vol. 37, no. 4, pp. 327-331, 1945.

[48] M. H. Jellinek and I. Fankuchen, "X-ray diffraction examination of Gamma alumina," Industrial \& Engineering Chemistry, vol. 37, no. 2, pp. 158-163, 1945.

[49] A. L. Patterson, "The diffraction of X-rays by small crystalline particles," Physical Review, vol. 56, no. 10, pp. 972-977, 1939.

[50] L. C. Roess and C. G. Shull, "X-ray scattering at small angles by finely-divided solids. II. Exact theory for random distributions of spheroidal particles," Journal of Applied Physics, vol. 18, no. 3, pp. 308-313, 1947.

[51] P. Mittelbach and G. Porod, "Zur Röntgenkleinwinkelstreuung kolloider Systeme: Die mittleren Durchschußlängen und die 
Kohärenzlänge eines kolloiden Systems; Kennzahlen zur Ermittlung von Teilchenform und Polydispersitätsgrad," KolloidZeitschrift \& Zeitschrift für Polymere, vol. 202, no. 1, pp. 40-49, 1965.

[52] B. Sjöberg, "Small-angle X-ray investigation of the equilibria between copper(II) and glycyl-1-histidylglycine in water solution. A method for analysing polydispersed systems," Journal of Applied Crystallography, vol. 7, no. 2, pp. 192-199, 1974.

[53] P. Schmidt, "The small angle X-ray scattering from polydisperse solutions of ellipsoidal particles," Acta Crystallographica, vol. 11, no. 10, pp. 674-676, 1958.

[54] Y. Mori, M. Furukawa, T. Hayashi, and K. Nakamura, "Size distribution of gold nanoparticles used by small angle X-ray scattering," Particulate Science and Technology, vol. 24, no. 1, pp. 97-103, 2006.

[55] M. L. Lavčević and A. Turković, "The measurements of particle/crystallite size in nanostructured $\mathrm{TiO}_{2}$ films by SAXS/WAXD method," Scripta Materialia, vol. 46, no. 7, pp. 501-505, 2002.

[56] L. C. Roess, "A simple method of obtaining a particle mass distribution by inverting the X-ray intensity scattered at small angles," The Journal of Chemical Physics, vol. 14, no. 11, pp. 695697, 1946.

[57] J. Riseman, "Particle-size distribution from small-angle X-ray scattering," Acta Crystallographica, vol. 5, no. 2, pp. 193-196, 1952.

[58] V. Luzzati, "Sur deux problemes relatifs a la diffusion des rayons $\mathrm{X}$ aux petits angles: determination de la distribution des masses et correction du polychromatisme," Acta Crystallographica, vol. 10, no. 1, pp. 33-34, 1957.

[59] J. H. Letcher and P. W. Schmidt, "Small-angle X-ray scattering determination of particle-diameter distributions in polydisperse suspensions of spherical particles," Journal of Applied Physics, vol. 37, no. 2, pp. 649-655, 1966.

[60] O. L. Brill and P. W. Schmidt, "Small-angle X-ray-scattering determination of diameter distributions," Journal of Applied Physics, vol. 39, no. 5, pp. 2274-2281, 1968.

[61] O. L. Brill, C. G. Weil, and P. W. Schmidt, "Determination of particle-diameter distributions in silica and gold suspensions," Journal of Colloid And Interface Science, vol. 27, no. 3, pp. 479492, 1968.

[62] G. Walter, R. Kranold, T. Gerber, J. Baldrian, and M. Steinhart, "Particle size distribution from small-angle X-ray scattering data," Journal of Applied Crystallography, vol. 18, no. 4, pp. 205213, 1985.

[63] I. S. Fedorova and V. B. Emelyanov, "Solution of inverse problems of scattering in the Rayleigh-Debye approximation. II. Determination of diameter distribution functions of thin spherical shells," Journal of Colloid and Interface Science, vol. 59, no. 1, pp. 106-112, 1977.

[64] I. S. Fedorova, "Solution of inverse scattering problems in the Rayleigh-Debye approximation. I. Determination of the diameter distribution of assemblies of long cylinders," Journal of Colloid and Interface Science, vol. 59, no. 1, pp. 98-105, 1977.

[65] P. W. Schmidt, V. B. Emel'yanov, and I. S. Fedorova, "Solution of inverse scattering problems in the Rayleigh-Debye approximation. III. Calculation of the length distribution for assemblies of thin cylinders," Journal of Colloid and Interface Science, vol. 67, no. 2, pp. 226-233, 1978.

[66] P. W. Schmidt, I. S. Fedorova, and V. B. Emel'yanov, "Solution of inverse scattering problems in the Rayleigh-Debye approximation. IV. Calculation of the diameter distribution function for assemblies of thin circular discs," Journal of Colloid and Interface Science, vol. 67, no. 2, pp. 234-239, 1978.

[67] I. S. Fedorova, "Solution of inverse scattering problems in the Rayleigh-Debye approximation. V. Determination of the diameter distribution functions of helical structures," Journal of Colloid and Interface Science, vol. 73, no. 1, pp. 208-211, 1980.

[68] I. S. Fedorova and P. W. Schmidt, "A general analytical method for calculating particle-dimension distributions from scattering data," Journal of Applied Crystallography, vol. 11, no. 5, pp. 405411, 1978.

[69] M. Mulato and I. Chambouleyron, "Small-angle X-ray and neutron scattering of polydisperse systems: determination of the scattering-particle-size distribution," Journal of Applied Crystallography, vol. 29, no. 1, pp. 29-36, 1996.

[70] M. Mulato, D. Tygel, and I. Chambouleyron, "On the retrieval of particle size distributions from small-angle scattering data: the influence of statistical data dispersion," Journal of Applied Crystallography, vol. 31, no. 2, pp. 149-153, 1998.

[71] R. W. Hendricks, J. Schelten, and W. Schmatz, "Studies of voids in neutron-irradiated aluminium single crystals: II. small-angle neutron scattering," Philosophical Magazine, vol. 30, no. 4, pp. 819-837, 1974.

[72] C. Vonk, "On two methods of determination of particle size distribution functions by means of small-angle X-ray scattering," Journal of Applied Crystallography, vol. 9, no. 6, pp. 433-440, 1976.

[73] A. N. Tichonov and Y. Arsenin, Solution of Ill Posed Problems, WH Winston, Washington, DC, USA, 1977.

[74] O. Glatter, "Determination of particle-size distribution functions from small-angle scattering data by means of the indirect transformation method," Journal of Applied Crystallography, vol. 13, no. 1, pp. 7-11, 1980.

[75] D. I. Svergun, A. V. Semenyuk, and L. A. Feigin, "Small-anglescattering-data treatment by the regularization method," Acta Crystallographica Section A, vol. 44, no. 3, pp. 244-250, 1988.

[76] H. G. Krauthaeuser, W. Heitmann, A. Kops, and G. Nimtz, "Small-angle $\mathrm{X}$-ray scattering analysis of particle-size distributions of mesoscopic metallic systems with consideration of the particle form factor," Journal of Applied Crystallography, vol. 27, no. 4, pp. 558-562, 1994.

[77] P. Moore, "Small-angle scattering. Information content and error analysis," Journal of Applied Crystallography, vol. 13, no. 2, pp. $168-175,1980$.

[78] D. Tatchev and R. Kranold, "Maximum-entropy method as a routine tool for determination of particle size distributions by small-angle scattering," Journal of Applied Crystallography, vol. 37, no. 1, pp. 32-39, 2004.

[79] V. Goertz, N. Dingenouts, and H. Nirschl, "Comparison of nanometric particle size distributions as determined by SAXS, TEM and analytical ultracentrifuge," Particle and Particle Systems Characterization, vol. 26, no. 1-2, pp. 17-24, 2009.

[80] J. Skilling, Maximum Entropy and Bayesian Methods, Kluwer Academic Publisher, Dordrecht, The Netherlands, 1989.

[81] J. A. Potton, G. J. Daniell, A. D. Eastop et al., "Ferrofluid particle size distributions from magnetisation and small angle neutron scattering data," Journal of Magnetism and Magnetic Materials, vol. 39, no. 1-2, pp. 95-98, 1983.

[82] S. Hansen and J. S. Pedersen, "Comparison of three different methods for analysing small-angle scattering data," Journal of Applied Crystallography, vol. 24, no. 5, pp. 541-548, 1991. 
[83] S. Hansen, "Bayesian estimation of hyperparameters for indirect Fourier transformation in small-angle scattering," Journal of Applied Crystallography, vol. 33, no. 6, pp. 1415-1421, 2000.

[84] C. S. Tsao and T. L. Lin, "Analysis of small-angle scattering data from spherical particles by both the indirect transform method and the maximum-entropy method," Journal of Applied Crystallography, vol. 30, no. 3, pp. 353-361, 1997.

[85] T.-L. Lin and C.-S. Tsao, "The analysis of small-angle scattering data from polydisperse rodlike particles by indirect transform and maximum-entropy methods," Journal of Applied Crystallography, vol. 29, no. 2, pp. 170-177, 1996.

[86] P. R. Jemian and A. J. Allen, "The effect of the shape function on small-angle scattering analysis by the maximum-entropy method," Journal of Applied Crystallography, vol. 27, no. 5, pp. 693-702, 1994.

[87] H. G. Krauthäuser, "Deducing material properties from indirect measurements," Physica A, vol. 211, no. 2-3, pp. 317-326, 1994.

[88] H. G. Krauthäuser, W. Lennartz, and G. Nimtz, "Real-space distributions from small-angle scattering data: structure interference method versus indirect transformation method," Journal of Applied Crystallography, vol. 29, no. 1, pp. 7-15, 1996.

[89] S. Martelli and P. E. Di Nunzio, "Particle size distribution of nanospheres by Monte Carlo fitting of small angle X-ray scattering curves," Particle \& Particle Systems Characterization, vol. 19, no. 4, pp. 247-255, 2002.

[90] P. E. Di Nunzio, S. Martelli, and R. R. Bitti, "Use of Monte Carlo methods in characterizing nanostructured materials by wideand small-angle x-ray scattering," Journal of Dispersion Science and Technology, vol. 25, no. 4, pp. 491-501, 2004.

[91] P. E. Di Nunzio, S. Martelli, and R. Ricci Bitti, "A Monte Carlo estimate of crystallite-size and microstrain distribution functions from X-ray line broadening," Journal of Applied Crystallography, vol. 28, no. 2, pp. 146-159, 1995.

[92] B. R. Pauw, J. S. Pedersen, S. Tardif, M. Takatab, and B. B. Iversen, "Improvements and considerations for size distribution retrieval from small-angle scattering data by monte-carlo methods," Journal of Applied Crystallography Short Communications, vol. 46, no. 2, pp. 365-371, 2012.

[93] G. Tóth, "Simultaneous Monte Carlo determination of particle size distribution and pair-correlation function of spherical colloids from a diffraction experiment," Langmuir, vol. 15, no. 20, pp. 6718-6723, 1999.

[94] G. Tóth, "Monte Carlo determination of the radii and the paircorrelation function of spherical colloids," Physica B, vol. 276278, pp. 404-405, 2000.

[95] J. Teixeira, "Small-angle scattering by fractal systems," Journal of Applied Crystallography, vol. 21, no. 6, pp. 781-785, 1998.

[96] N. K. Ailawadi, "Equilibrium theories of simple liquids," Physics Reports, vol. 57, no. 4, pp. 241-306, 1980.

[97] E. Kaler, "Small-angle scattering from colloidal dispersions," Journal of Applied Crystallography, vol. 21, no. 6, pp. 729-736, 1988.

[98] J. T. Schelten and W. Schmatz, "Multiple-scattering treatment for small-angle scattering problems," Journal of Applied Crystallography, vol. 13, no. 4, pp. 385-390, 1980.

[99] N. F. Berk and K. A. Hardman-Rhyne, "Analysis of SAS data dominated by multiple scattering," Journal of Applied Crystallography, vol. 21, no. 6, pp. 645-651, 1998.

[100] J. P. Hansen and I. R. McDonald, Theory of Simple Liquids, Elsevier Academic Press, London, UK, 2006.
[101] L. Belloni, Interacting Monodispersed and Polydispersed Spheres, Elsvier, Amsterdam, The Netherlands, 1991.

[102] R. J. Baxter, "Percus-Yevick equation for hard spheres with surface adhesion," The Journal of Chemical Physics, vol. 49, no. 6, pp. 2770-2774, 1968.

[103] E. J. W. Verwey and J. T. G. Overbeek, Theory of the Stability of Lyophobic Colloids, Courier Dover Publications, Mineola, NY, USA, 1999.

[104] J. K. Percus and G. J. Yevick, "Analysis of classical statistical mechanics by means of collective coordinates," Physical Review, vol. 110, no. 1, pp. 1-13, 1958.

[105] J. B. Hayter and J. Penfold, "An analytic structure factor for macroion solutions," Molecular Physics, vol. 42, no. 1, pp. 109$118,1981$.

[106] B. Weyerich, J. Brunner-Popela, and O. Glatter, "Small-angle scattering of interacting particles. II. Generalized indirect Fourier transformation under consideration of the effective structure factor for polydisperse systems," Journal of Applied Crystallography, vol. 32, no. 2, pp. 197-209, 1999.

[107] L. Blum and G. Stell, "Polydisperse systems. I. Scattering function for polydisperse fluids of hard or permeable spheres," The Journal of Chemical Physics, vol. 71, no. 1, pp. 42-46, 1979.

[108] C. Robertus, W. H. Philipse, J. G. H. Joosten, and Y. K. Levine, "Solution of the Percus-Yevick approximation of the multicomponent adhesive spheres system applied to the small angle X-ray scattering from microemulsions," The Journal of Chemical Physics, vol. 90, no. 8, pp. 4482-4490, 1989.

[109] L. Blum, "Solution of the Ornstein-Zernike equation for a mixture of hard ions and Yukawa closure," Journal of Statistical Physics, vol. 22, no. 6, pp. 661-672, 1980.

[110] M. Kotlarchyk and S.-H. Chen, "Analysis of small angle neutron scattering spectra from polydisperse interacting colloids," The Journal of Chemical Physics, vol. 79, no. 5, pp. 2461-2469, 1983.

[111] J. S. Pedersen, "Determination of size distributions from smallangle scattering data for systems with effective hard-sphere interactions," Journal of Applied Crystallography, vol. 27, no. 4, pp. 595-608, 1994.

[112] S. Hansen, "Monte Carlo estimation of the structure factor for hard bodies in small-angle scattering," Journal of Applied Crystallography, vol. 45, no. 3, pp. 381-388, 2012.

[113] S. Hansen, "The structure factor in small-angle scattering and the effect of deviation from spherical symmetry," Journal of Applied Crystallography, vol. 44, no. 2, pp. 265-271, 2011.

[114] J. Brunner-Popela and O. Glatter, "Small-angle scattering of interacting particles. I. Basic principles of a global evaluation technique," Journal of Applied Crystallography, vol. 30, no. 4, pp. 431-442, 1997.

[115] H. M. A. Ehmann, S. Spirk, A. Doliska et al., "Generalized indirect Fourier transformation as a valuable tool for the structural characterization of aqueous nanocrystalline cellulose suspensions by small angle X-ray scattering," Langmuir, vol. 29, no. 11, pp. 3740-3748, 2013.

[116] J. Brunner-Popela, R. Mittelbach, R. Strey, K.-V. Schubert, E. W. Kaler, and O. Glatter, "Small-angle scattering of interacting particles. III. $\mathrm{D}_{2} \mathrm{O}-\mathrm{C}_{12} \mathrm{E}_{5}$ mixtures and microemulsions with $n$ octane," The Journal of Chemical Physics, vol. 110, no. 21, pp. 10623-10632, 1999.

[117] N. Lutterbach, H. Versmold, V. Reus, L. Belloni, and T. Zemb, "Charge-stabilized liquidlike ordered binary colloidal suspensions. 1. Ultra-small-angle X-ray scattering characterization," Langmuir, vol. 15, no. 2, pp. 337-344, 1999. 
[118] G.-W. Lee, K. S. Jin, J. Kim et al., "Small angle X-ray scattering studies on structures of alkylthiol stabilized-silver nanoparticles in solution," Applied Physics A, vol. 91, no. 4, pp. 657-661, 2008. 

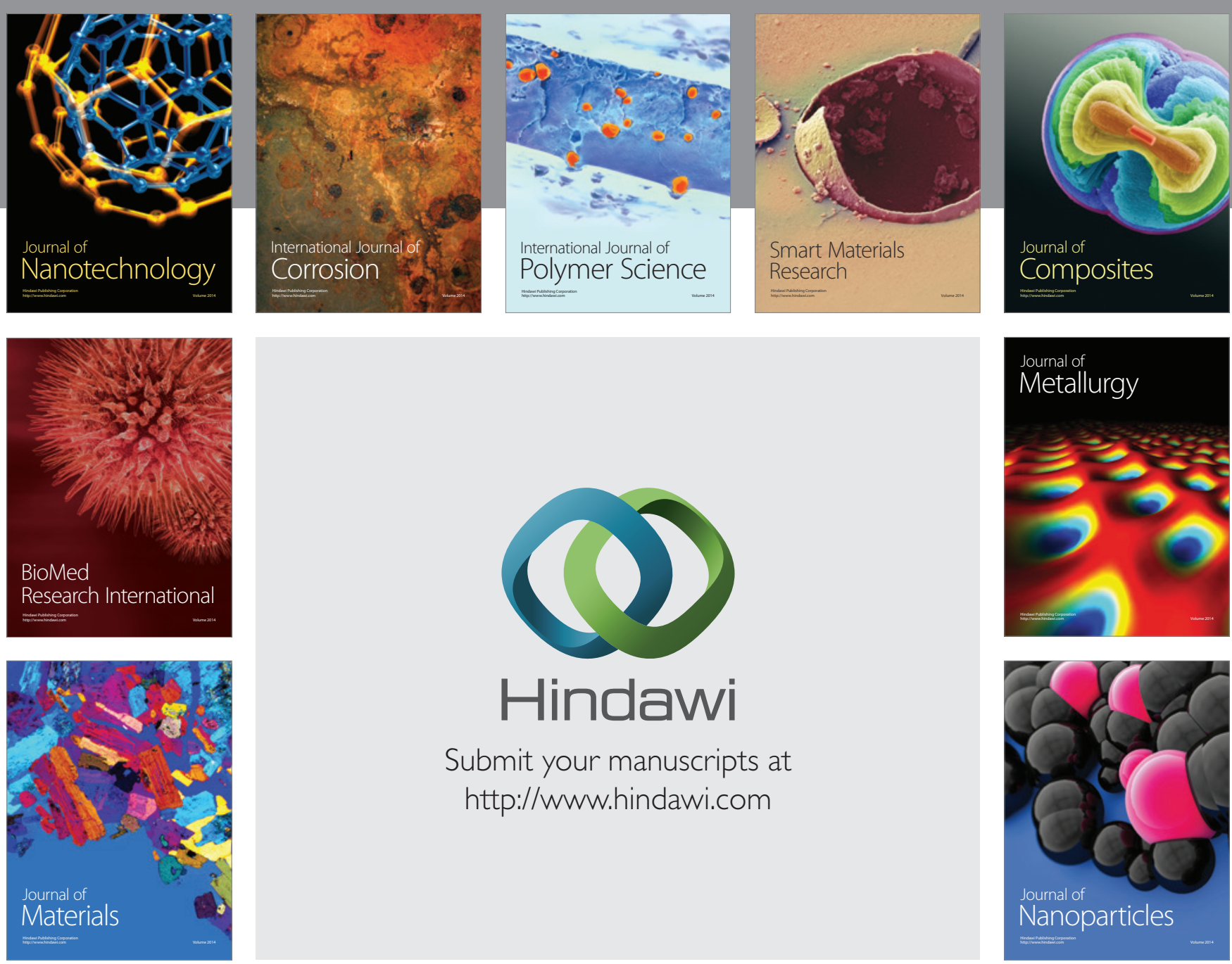

Submit your manuscripts at http://www.hindawi.com
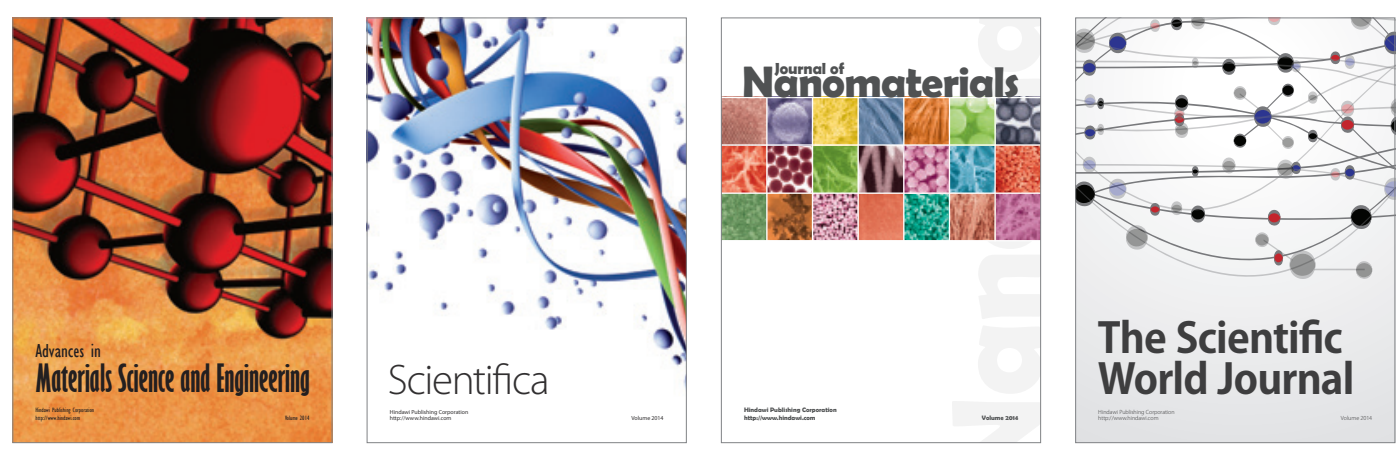

\section{The Scientific World Journal}
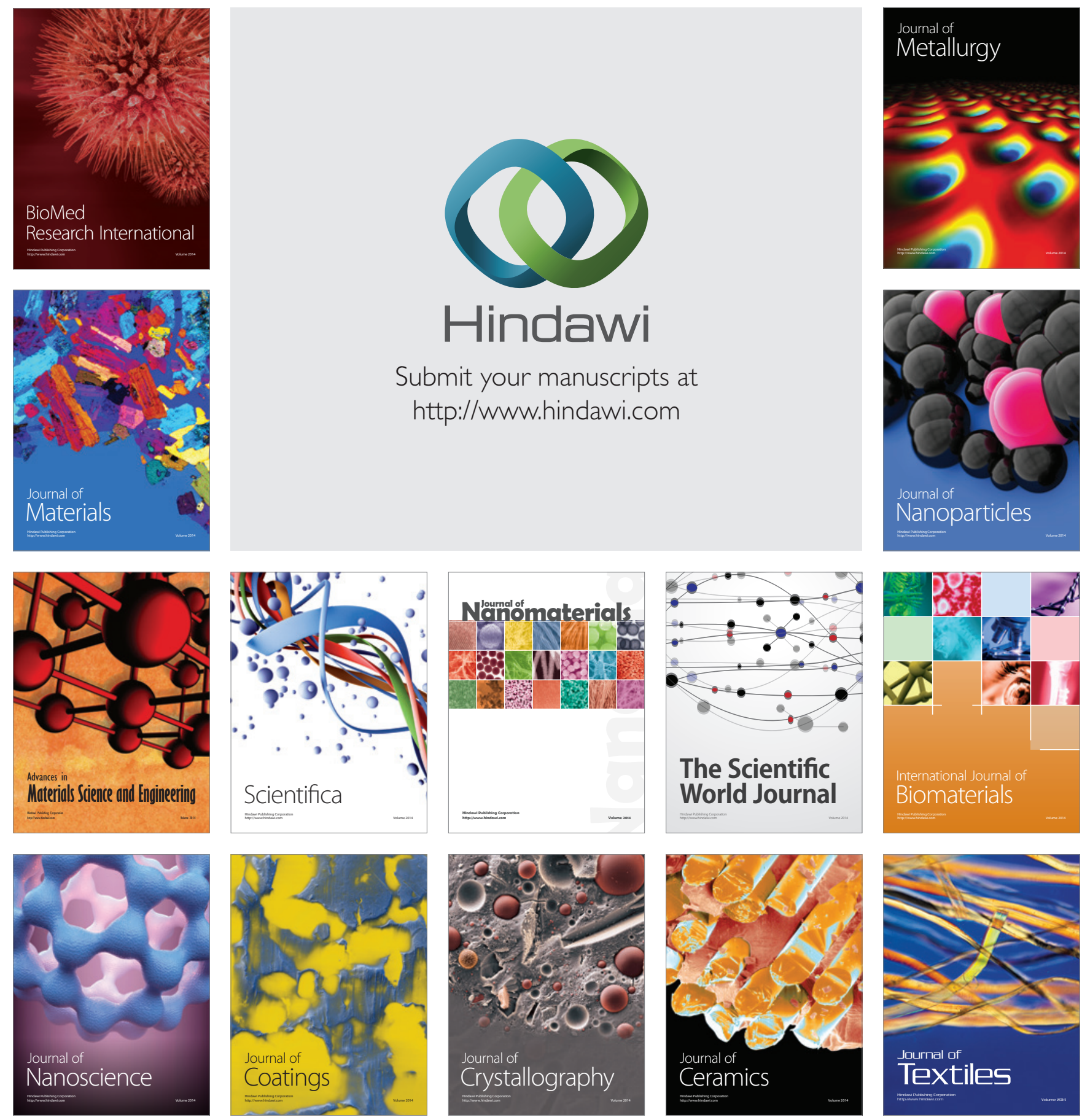